Pure and Applied Mathematics Quarterly

Volume 7, Number 4

(Special Issue:

In memory of Eckart Viehweg)

1371-1393, 2011

\title{
Anticanonical Divisors and Curve Classes on Fano Manifolds
}

\author{
Andreas Höring and Claire Voisin
}

To Eckart Viehweg

\begin{abstract}
It is well known that the Hodge conjecture with rational coefficients holds for degree $2 n-2$ classes on complex projective $n$-folds. In this paper we study the more precise question if on a rationally connected complex projective $n$-fold the integral Hodge classes of degree $2 n-2$ are generated over $\mathbb{Z}$ by classes of curves. We combine techniques from the theory of singularities of pairs on the one hand and infinitesimal variation of Hodge structures on the other hand to give an affirmative answer to this question for a large class of manifolds including Fano fourfolds. In the last case, one step in the proof is the following result of independent interest: There exist anticanonical divisors with isolated canonical singularities on a smooth Fano fourfold.
\end{abstract}

Keywords: Canonical singularities, Fano varieties, curve classes, variation of Hodge structure.

\section{INTRODUCTION}

The Hodge conjecture states that the space $H d g^{2 i}(X)$ of rational Hodge classes on a smooth projective complex variety $X$ is generated over $\mathbb{Q}$ by classes of algebraic cycles of codimension $i$ on $X$. This conjecture is known to be wrong for integral coefficients instead of rational coefficients ([1], [12]). We will focus in this paper

Received: Sept. 15, 2010; Revised: Jan. 12, 2011. 
on Hodge classes of degree $2 n-2, n=\operatorname{dim} X$ (or "curve classes") for which the Hodge conjecture is known to hold with rational coefficients. As remarked in [23], this case is particularly interesting as it leads to a birational invariant of $X$, namely the finite group

$$
Z^{2 n-2}(X):=H d g^{2 n-2}(X, \mathbb{Z}) /\langle[Z], \operatorname{codim} Z=n-1\rangle,
$$

where

$$
H d g^{2 n-2}(X, \mathbb{Z}):=\left\{\alpha \in H^{2 n-2}(X, \mathbb{Z}), \alpha_{\mathbb{C}} \in H^{n-1, n-1}(X)\right\} .
$$

Kollár's counterexamples show that this group can be non trivial starting from $n=3$ and are strikingly simple: He considers smooth hypersurfaces $X$ of degree $d$ in $\mathbb{P}^{4}$. The cohomology group $H^{4}(X, \mathbb{Z})$ is then isomorphic to $\mathbb{Z}$, with generator $\alpha$ of degree 1 with respect to the hyperplane class.

1.1. Theorem.(Kollár, [12]) Assume that for some integer $p$ coprime to $6, p^{3}$ divides $d$. Then for very general such $X$, any curve $C \subset X$ has degree divisible by $p$. Hence the class $\alpha$ is not algebraic, (that is, is not the class of an algebraic cycle).

Kollár's examples are hypersurfaces of general type (and in fact, hypersurfaces with negative or trivial canonical class contain a line, whose cohomology class is the generator $\alpha$ ). It was proved more generally in [27] that the Kodaira dimension plays an important role in the study of the group $Z^{4}(X)$, when $\operatorname{dim} X=3$ :

1.2. Theorem. (Voisin 2006) Let $X$ be a smooth projective threefold. Assume that $X$ is either uniruled or Calabi-Yau. Then the Hodge conjecture holds for integral Hodge classes on $X$, that is, the group $Z^{4}(X)$ is trivial.

The assumptions on the Kodaira dimension are "essentially optimal". (Note however that in this theorem, the assumption in the Calabi-Yau case is not actually an assumption on the Kodaira dimension, since we do not know whether, in the theorem above, it could be replaced by the weaker assumption that the Kodaira dimension is 0.) Indeed, mimicking Starr's degeneration argument in [24], one can show that a very general hypersurface $X$ in $\mathbb{P}^{1} \times \mathbb{P}^{3}$ of bidegree $(3,4)$, which has Kodaira dimension 1, has a non trivial group $Z^{4}(X)$ : More precisely, the Lefschetz hyperplane section theorem shows that the group $H^{4}(X, \mathbb{Z})=H_{2}(X, \mathbb{Z})$ consists of Hodge classes and surjects via the Gysin map $p r_{1 *}$ onto $\mathbb{Z}=H_{2}\left(\mathbb{P}^{1}, \mathbb{Z}\right)$. On the other hand, one can show that for very general such $X$, any curve $C \subset X$ 
has even degree over $\mathbb{P}^{1}$ so that the images via the Gysin map $p r_{1 *}$ of algebraic classes do not generate $H_{2}\left(\mathbb{P}^{1}, \mathbb{Z}\right)$ over $\mathbb{Z}$.

In dimension 4 and higher, the uniruledness assumption does not say anything on the group $Z^{2 n-2}(X)$. Indeed, start from one of the Kollár's examples $X_{1}$, and consider the uniruled fourfold $X:=X_{1} \times \mathbb{P}^{1}$. The degree 6 integral Hodge class

$$
\beta:=p r_{1}^{*} \alpha \cup p r_{2}^{*}[p t],
$$

is not algebraic on $X$, since otherwise $\operatorname{pr}_{2 *} \beta=\alpha$ would be algebraic on $X_{1}$. We are thus led to consider the more restricted class of rationally connected projective $n$-folds $X$. Note that for such $X$, the Hodge structure on $H^{2 n-2}(X, \mathbb{Q})$ is trivial, being dual to the Hodge structure on $H^{2}(X, \mathbb{Q})$, which is trivial because $H^{0}\left(X, \Omega_{X}^{2}\right)=0$. Hence all rational cohomology classes of degree $2 n-2$ on such $X$ are Hodge. The following question was raised in [23, section 2]:

1.3. Question. Is the integral cohomology of degree $2 n-2$ of a rationally connected manifold of dimension $n$ generated over $\mathbb{Z}$ by classes of curves?

We will prove in this paper the following result, which answers positively this question for certain Fano manifolds, and also for certain projective manifolds $X$ for which $-K_{X}$ is only assumed to be 1 -ample. Recall from [22] that a vector bundle $\mathcal{E}$ on $X$ is said to be 1-ample if some multiple $\mathcal{O}_{\mathbb{P}(E)}(l), l>0$ of the tautological line bundle on $\mathbb{P}(\mathcal{E})$ is globally generated, and the fibers of the morphism $\mathbb{P}(\mathcal{E}) \rightarrow \mathbb{P}^{N}$ given by sections of $\mathcal{O}_{\mathbb{P}(E)}(l)$ are at most 1-dimensional.

1.4. Theorem. Let $X$ be a rationally connected projective manifold of dimension $n \geq 4$. Assume that there exists a 1-ample vector bundle $\mathcal{E}$ of rank $n-3$ on $X$ such that det $\mathcal{E}=-K_{X}$ and $\mathcal{E}$ has a transverse section whose zero set $Y$ has isolated canonical singularities. Then the group $H^{2 n-2}(X, \mathbb{Z})$ is generated over $\mathbb{Z}$ by classes of curves (equivalently, $Z^{2 n-2}(X)=0$ ).

The property that the 3 -fold $Y$ has isolated singularities will be crucial for the proof of this Theorem : it assures that if we consider a sufficiently general ample divisor $\Sigma \subset Y$, it does not meet the singular locus of $Y$. We then generalize the methods from [27], based on the study of the infinitesimal variation of Hodge structure of these surfaces $\Sigma$, to conclude.

If $\mathcal{E}$ is globally generated, a generic section of $\mathcal{E}$ is transverse with smooth vanishing locus; hence we get the following corollary: 
1.5. Corollary. Let $X$ be a rationally connected projective manifold of dimension $n \geq 4$. Assume that there exists a globally generated 1 -ample vector bundle $\mathcal{E}$ of rank $n-3$ on $X$ such that det $\mathcal{E}=-K_{X}$. Then the group $H^{2 n-2}(X, \mathbb{Z})$ is generated over $\mathbb{Z}$ by classes of curves.

Let us now give a class of examples covered by Theorem 1.4, but not by Corollary 1.5: a Fano manifold of dimension $n \geq 4$ has index $n-3$ if there exists an ample Cartier divisor $H$ (called the fundamental divisor) such that $-K_{X} \simeq(n-3) H$. For these manifolds we will prove the following result:

1.6. Theorem. Let $X$ be a smooth Fano manifold of dimension $n \geq 4$ and index $n-3$. Suppose moreover that the fundamental divisor satisfies $h^{0}(X, H) \geq n-2$. Let $Y$ be the zero locus of a general section of the vector bundle $\mathcal{E}=H^{\oplus n-3}$. Then $Y$ has dimension 3 (i.e. the general section is transverse) and has isolated canonical singularities.

We expect the technical condition $h^{0}(X, H) \geq n-2$ to be automatically satisfied and prove this in low dimension (cf. also [5]). In particular we obtain the following unconditional theorem concerning Question 1.3 in the case of Fano manifolds of dimension at most five.

1.7. Theorem. i) Let $X$ be a smooth Fano fourfold. Then a general anticanonical divisor $Y \in\left|-K_{X}\right|$ has canonical isolated singularities.

ii) $X$ being as above, the group $H^{6}(X, \mathbb{Z})$ is generated over $\mathbb{Z}$ by classes of curves (equivalently, $Z^{6}(X)=0$ ).

iii) Let $X$ be a smooth Fano fivefold of index 2. Then the group $H^{8}(X, \mathbb{Z})$ is generated over $\mathbb{Z}$ by classes of curves (equivalently, $Z^{8}(X)=0$ ).

1.8. Remark. According to Kollár [15], Iskovskikh asked whether curve classes on Fano manifolds $X$ are generated over $\mathbb{Z}$ by classes of rational curves. This question is of a different nature and leads to another birationally invariant group associated to $X$, namely the group of curve classes on $X$ modulo the subgroup generated by classes of rational curves in $X$. Kollár (see [14, Thm.3.13]) proves that for rationally connected manifolds $X$, curve classes on $X$ are generated over $\mathbb{Q}$ by classes of rational curves. In other words, the group introduced above is of torsion. The method used here does not give any insight on the Iskovskikh question, that is whether this torsion group is zero or not for Fano manifolds. 
Note that our result in Theorem 1.7, i) on the anticanonical divisor is optimal, i.e. it is easy to construct examples of Fano fourfolds without any smooth anticanonical divisor (cf. Example 2.12). In particular it is not possible to prove the statement ii) of Theorem 1.7 simply by applying the Lefschetz hyperplane theorem to $Y$ and using the Calabi-Yau case of Theorem 1.2.

Let us explain the proof of Theorem 1.6 if $X$ is a fourfold. Based on subadjunction techniques and effective non-vanishing results, Kawamata has shown in [10] that a general anticanonical divisor $Y$ is a Calabi-Yau threefold with at most canonical singularities, in particular the singular locus of $Y$ has dimension at most one. We will argue by contradiction and suppose that a general $Y$ is singular along a curve $C$. A priori the curve $C$ depends on $Y$, but we show that if we fix a pencil spanned by two general elements $Y_{1}, Y_{2}$ in $\left|-K_{X}\right|$, all the elements of the pencil are singular along the same curve $C$. In particular the pair $\left(X, Y_{1}+Y_{2}\right)$ is not log-canonical along the curve $C$, by inversion of adjunction this contradicts a result of Kawamata on ample divisors on Calabi-Yau threefolds [10, Prop.4.2].

If we apply the same argument to Fano threefolds we obtain a new proof of Shokurov's theorem.

1.9. Theorem. [21] Let $X$ be a smooth Fano threefold. If $Y \in\left|-K_{X}\right|$ is a general element, it is smooth.

Earlier proofs of this statement used Saint Donat's study of linear systems on K3 surfaces [20] which so far has no analogue in higher dimension. By contrast our strategy of proof generalizes immediately to arbitrary dimension and shows the following: if Kawamata's nonvanishing conjecture [10, Conj.2.1] is true, the singular locus of a general anticanonical divisor on any Fano manifold has codimension at least three (which is the optimal bound).

Let us conclude this introduction with a comment on assumptions in Theorem 1.4: in the proof of Theorem 1.4 we will only use that $-K_{X}$ is 1 -ample and the cohomology group $H^{1}\left(X, \mathcal{O}_{X}\right)$ vanishes. This seems to be weaker than supposing that $X$ is rationally connected and $-K_{X}$ is 1 -ample, but in fact these conditions are equivalent:

1.10. Proposition. Let $X$ be a projective manifold such that $-K_{X}$ is 1-ample and $H^{1}\left(X, \mathcal{O}_{X}\right)=0$. Then $X$ is rationally connected. 
Proof. Since $-K_{X}$ is nef with numerical dimension $n-1$, we have $H^{i}\left(X, \mathcal{O}_{X}\right)=0$, for $i>1[3,6.13]$. Hence we have $\chi\left(X, \mathcal{O}_{X}\right)=1$. Furthermore $X$ has no finite étale cover, because an étale cover $X^{\prime} \rightarrow X$ of degree $m>1$ would also satisfy the condition that $-K_{X^{\prime}}$ is 1 -ample, which implies as above that $H^{i}\left(X^{\prime}, \mathcal{O}_{X^{\prime}}\right)=0$, for $i>1$. Thus $\chi\left(X^{\prime}, \mathcal{O}_{X^{\prime}}\right) \leq 1$, which contradicts $\chi\left(X^{\prime}, \mathcal{O}_{X^{\prime}}\right)=m \chi\left(X, \mathcal{O}_{X}\right)=m$.

According to [4], it follows that $X$ is a product of Calabi-Yau varieties, symplectic holomorphic varieties and varieties $W$ such that $H^{0}\left(W, \Omega_{W}^{\otimes l}\right)=0, l>0$. The first two types can not occur because $H^{0}\left(X, \Omega_{X}^{l}\right)=0, l>0$. It follows that $H^{0}\left(X, \Omega_{X}^{\otimes l}\right)=0, l>0$.

Consider now the maximal rationally connected fibration $\psi: X \rightarrow B$ of $X$ (cf. [13]). If $X$ is not rationally connected, $B$ is not uniruled by [6], and thus the Kodaira dimension of $B$ is 0 , according to [28]. But then, if $b:=\operatorname{dim} B>0$, there is a non zero section of $K_{B}^{\otimes m}$ for some $m>0$, which gives a non zero section of $\Omega_{X}^{\otimes b m}$ and provides a contradiction.

We dedicate this paper to the memory of Eckart Viehweg, who contributed in a major way to both birational geometry and Hodge theory.

\section{AntiCANONICAL Divisors}

While anticanonical divisors on Fano threefolds (and Fano $n$-folds of index $n-2$ ) are well understood [21, 16], our knowledge on higher-dimensional Fano manifolds remains rather limited. A first step was done by Kawamata:

2.11. Theorem. [10, Thm.5.2] Let $X$ be a Fano fourfold with at most canonical Gorenstein singularities. Then the anticanonical system is not empty. If $Y \in$ $\left|-K_{X}\right|$ is a general element, it is a Calabi-Yau threefold with at most canonical singularities.

In view of this statement and Shokurov's theorem 1.9 one might hope that for a smooth Fano fourfold the general anticanonical divisor $Y$ is also smooth. Here is an easy counterexample which shows that such an $Y$ might even not be $\mathbb{Q}$-Cartier:

2.12. Example. Let $S$ be the blow-up of $\mathbb{P}^{2}$ in eight points in general position. Then $S$ is a Fano surface whose anticanonical system has exactly one base point 
which we denote by $p$. Set $X:=S \times S$ and $S_{i}:=p_{i}^{-1}(p)$ where $p_{i}$ is the projection on the $i$-th factor. Then $X$ is a smooth Fano fourfold and

$$
B s\left|-K_{X}\right|=S_{1} \cup S_{2} .
$$

Let $Y \in\left|-K_{X}\right|$ be a general element, then $B s\left|-K_{X}\right| \subset Y$, so the surfaces $S_{1}, S_{2}$ are Weil divisors in $Y$. If they were $\mathbb{Q}$-Cartier, their intersection $S_{1} \cap S_{2}$ would have dimension at least one, yet we have $S_{1} \cap S_{2}=(p, p)$. Thus the variety $Y$ is not $\mathbb{Q}$-factorial.

Very recently Floris generalised Theorem 2.11 to Fano varieties of index $n-3$ :

2.13. Theorem. [5] Let $X$ be a smooth Fano manifold of dimension $n \geq 4$ and index $n-3$. Suppose moreover that the fundamental divisor satisfies $h^{0}(X, H) \geq$ $n-2$. Then there exists a sequence

$$
X \supsetneq Z_{1} \supsetneq Z_{2} \supsetneq \ldots \supsetneq Z_{n-3}
$$

such that $Z_{i+1} \in|H|_{Z_{i}} \mid$ is a normal variety of dimension $n-i$ with at most canonical singularities.

2.A. Proof of the structure results. Let us recall that if $X$ is a normal variety and $D=\sum_{i} d_{i} D_{i}$ with $0<d_{i} \leq 1$ for all $i$ an effective $\mathbb{Q}$-divisor on $X$ such that $K_{X}+D$ is $\mathbb{Q}$-Cartier, the pair $(X, D)$ is log-canonical if for every birational morphism $\mu: X^{\prime} \rightarrow X$ from a normal variety $X^{\prime}$ we can write

$$
K_{X^{\prime}}+D^{\prime}=\mu^{*}\left(K_{X}+D\right)+\sum_{j} a_{j} E_{j}
$$

where $D^{\prime}$ is the strict transform of $D$ and $a_{j} \geq-1$ for all $j$.

Proof of Theorem 1.6. We will treat first the case of Fano fourfolds and in a second step apply Floris' result to prove the higher-dimensional case.

a) $\operatorname{dim} X=4$. Let $Y_{1}$ be a general element in $\left|-K_{X}\right|$ and consider the restriction sequence

$$
0 \rightarrow \mathcal{O}_{X} \rightarrow \mathcal{O}_{X}\left(-K_{X}\right) \rightarrow \mathcal{O}_{Y_{1}}\left(-K_{X}\right) \rightarrow 0 .
$$

Since $h^{1}\left(X, \mathcal{O}_{X}\right)=0$ we have a surjection

$$
H^{0}\left(X, \mathcal{O}_{X}\left(-K_{X}\right)\right) \rightarrow H^{0}\left(Y_{1}, \mathcal{O}_{Y_{1}}\left(-K_{X}\right)\right),
$$


so a general element in the linear system $\left|-K_{X}\right|_{Y_{1}} \mid$ is obtained by intersecting $Y_{1}$ with another general element $Y_{2} \in\left|-K_{X}\right|$. By [10, Prop.4.2] we know that for

$$
D:=Y_{1} \cap Y_{2} \subset\left|-K_{X}\right|_{Y_{1}} \mid
$$

general, the pair $\left(Y_{1}, D\right)$ is log-canonical. In particular $D$ is a reduced surface, so the singular locus of $D$ has dimension at most one. By Bertini's theorem we know that the singular locus $Y_{i, \text { sing }}$ of the divisor $Y_{i}$ is contained in the base locus of $\left|-K_{X}\right|$. In particular it is contained in $D$, but we can be more precise: since $D$ is a complete intersection cut out by the divisors $Y_{1}$ and $Y_{2}$ we have

$$
Y_{i, \text { sing }} \subset D_{\text {sing }} \quad \forall i=1,2 .
$$

Moreover by inversion of adjunction [11, Thm.7.5] the pair $\left(X, Y_{1}+Y_{2}\right)$ is logcanonical near the divisor $Y_{1}$.

We will now argue by contradiction and suppose that a general element in $\left|-K_{X}\right|$ is singular along a curve. Take a general element $Y^{\prime}$ in the pencil $<Y_{1}, Y_{2}>\subset$ $\left|-K_{X}\right|$ spanned by $Y_{1}$ and $Y_{2}$. Then we have

$$
Y^{\prime} \cap Y_{1}=Y_{2} \cap Y_{1}=D,
$$

so we see as above that

$$
Y_{\text {sing }}^{\prime} \subset D_{\text {sing }} \text {. }
$$

Since $Y^{\prime}$ varies in an infinite family with each member having a singular locus of dimension one and $D_{\text {sing }}$ has dimension at most one, there exists a curve $C \subset D_{\text {sing }}$ such that every general $Y^{\prime}$ is singular along $C$. By upper semicontinuity of the multiplicity this shows that both $Y_{1}$ and $Y_{2}$ are singular along the curve $C$.

Let now $\sigma: X^{\prime} \rightarrow X$ be the blow-up of $X$ along $C$. Since $X$ is smooth along $C$ we have

$$
K_{X^{\prime}}=\sigma^{*} K_{X}+2 E,
$$

where $E$ is the exceptional divisor. Moreover since $Y_{1}$ and $Y_{2}$ are singular along $C$ we have

$$
\sigma^{*} Y_{i}=Y_{i}^{\prime}+a_{i} E
$$

where $Y_{i}^{\prime}$ are the strict transforms of $Y_{i}$, and $a_{i} \geq 2$ for all $i=1,2$. Thus the pair $\left(X, Y_{1}+Y_{2}\right)$ is not log-canonical, a contradiction.

b) $\operatorname{dim} X$ arbitrary. By Theorem 2.13 there exists a sequence

$$
X \supsetneq Z_{1} \supsetneq Z_{2} \supsetneq \ldots \supsetneq Z_{n-3}
$$


such that $Z_{i+1} \in|H|_{Z_{i}} \mid$ is a normal variety of dimension $n-i$ with at most canonical singularities. In particular $Z_{n-3}$ is a Calabi-Yau threefold and arguing inductively we obtain surjective maps

$$
H^{0}(X, H) \rightarrow H^{0}\left(Z_{i}, \mathcal{O}_{Z_{i}}(H)\right) \quad \forall i=1, \ldots, n-3 .
$$

Thus $Z_{n-3}$ is obtained by intersecting $n-3$ general elements $Y_{1}, \ldots Y_{n-3} \in|H|$ and a general element in $|H|_{Z_{n-3}} \mid$ is obtained by intersecting $Z_{n-3}$ with another general element $Y_{n-2} \in|H|$. By [10, Prop.4.2] we see that for

$$
D:=Z_{n-3} \cap Y_{n-2} \subset|H|_{Z_{n-3}} \mid
$$

general, the pair $\left(Z_{n-3}, D\right)$ is log-canonical. In particular $D$ is a reduced surface, so the singular locus of $D$ has dimension at most one. Since $D$ is a complete intersection cut out by the divisors $Y_{1}, \ldots, Y_{n-2}$, we have

$$
Y_{i, \text { sing }} \subset D_{\text {sing }} \quad \forall i=1, \ldots, n-2 .
$$

Moreover by repeated use of inversion of adjunction the pair $\left(X, \sum_{i=1}^{n-2} Y_{i}\right)$ is logcanonical near $Z_{n-3}$.

We can now conclude as above : if a general element of $|H|$ is singular along a curve, we can use a general element $Y^{\prime}$ in the pencil $<Y_{n-3}, Y_{n-2}>\subset|H|$ to show that there exists a curve $C \subset D$ such that both $Y_{n-3}$ and $Y_{n-2}$ are singular along $C$. In particular the divisor $\sum_{i=1}^{n-2} Y_{i}$ has multiplicity $n$ along $C$, contradicting the log-canonicity of the pair $\left(X, \sum_{i=1}^{n-2} Y_{i}\right)$.

Proof of Theorem 1.7. By Theorem 1.4 and Theorem 1.6 we are left to show that $h^{0}(X, H) \geq n-2$ when $n \leq 5$ and $-K_{X}=(n-3) H$. This is established in [5, Thm.1.1] for $\operatorname{dim} X=4,5$; for the convenience of the reader we give two arguments in the fourfold case, one numerical and one of geometric nature:

a) By Kodaira vanishing we have $h^{0}\left(X,-K_{X}\right)=\chi\left(X,-K_{X}\right)$, so we know by the Riemann-Roch formula that

$$
h^{0}\left(X,-K_{X}\right)=\frac{1}{6}\left(-K_{X}\right)^{4}+\frac{1}{12}\left(-K_{X}\right)^{2} \cdot c_{2}(X)+\chi\left(X, \mathcal{O}_{X}\right) .
$$

Combining a recent result of Peternell [18, Thm.1.4] with [17, Thm.6.1] shows that $\left(-K_{X}\right)^{2} \cdot c_{2}(X)$ is non-negative. Since $\chi\left(X, \mathcal{O}_{X}\right)=1$ this gives the claim. 
b) By Theorem 2.11 the anticanonical system is not empty and contains a CalabiYau threefold $Y$ with canonical singularities. By [10, Prop.4.2] any ample divisor on such a threefold is effective. Since the restriction map $H^{0}\left(X,-K_{X}\right) \rightarrow$ $H^{0}\left(Y,-\left.K_{X}\right|_{Y}\right)$ is surjective, this implies the statement.

Proof of Theorem 1.9. Using Reid's method ([19, 0.5], cf. also [5, 4]) one sees that a general element $Y \in\left|-K_{X}\right|$ is a K3 surface with at most canonical singularities. The proof of [10, Prop.4.2] applies verbatim to K3 surfaces, so if $A$ is an ample Cartier divisor on a K3 surface $Y$ with at most canonical singularities and $D \in|A|$ a general element, then $(Y, D)$ is log-canonical.

Choose now $Y_{1}$ and $Y_{2}$ general elements in $\left|-K_{X}\right|$ such that the pair

$$
\left(Y_{1}, D:=Y_{1} \cap Y_{2}\right)
$$

is log-canonical. By inversion of adjunction this implies that the pair $\left(X, Y_{1}+Y_{2}\right)$ is log-canonical near $Y_{1}$. Since $Y_{1} \cap Y_{2}$ is a reduced curve, its singular locus is a union of points. Thus if a general element in $\left|-K_{X}\right|$ is singular, we can argue as in the proof of Theorem 1.6 to see that there exists a point $p \in D_{\text {sing }}$ such that both $Y_{1}$ and $Y_{2}$ are singular in $p$. But this implies that the divisor $Y_{1}+Y_{2}$ has multiplicity at least four in $p$. Since $X$ is a smooth threefold, the pair $\left(X, Y_{1}+Y_{2}\right)$ is not log-canonical in $p$, a contradiction.

2.B. Consequences of the canonical isolated singularities property. We will need later on the following vanishing results (Lemma 2.14 and Lemma 2.15): Let $Y$ be a Gorenstein projective threefold with isolated canonical singularities, such that $H^{2}\left(Y, \mathcal{O}_{Y}\right)=0$.

2.14. Lemma. For any desingularization $\tau: \widetilde{Y} \rightarrow Y$ of $Y$, one has

$$
H^{2}\left(\widetilde{Y}, \mathcal{O}_{\widetilde{Y}}\right)=\{0\}
$$

and $H^{0}\left(\widetilde{Y}, \Omega_{\widetilde{Y}}^{2}\right)=\{0\}$.

Proof. Indeed, the singularities of $Y$ are canonical, hence rational. Thus

$$
H^{2}\left(\widetilde{Y}, \mathcal{O}_{\widetilde{Y}}\right)=H^{2}\left(Y, \mathcal{O}_{Y}\right)=0
$$

By Hodge symmetry on $\tilde{Y}$, the vanishing of $H^{2}\left(\widetilde{Y}, \mathcal{O}_{\tilde{Y}}\right)$ implies the vanishing of $H^{0}\left(\widetilde{Y}, \Omega_{\widetilde{Y}}^{2}\right)$. 
We will need in the next section a stronger form of this vanishing result. We assume as above that $Y$ is a Gorenstein projective threefold with isolated canonical singularities, such that $H^{2}\left(Y, \mathcal{O}_{Y}\right)=0$. Let now $H$ be an ample line bundle on $Y$.

2.15. Lemma. For any sufficiently large and divisible $n$, and any $\Sigma \in|n H|$ contained in $Y_{\text {reg, }}$ one has

$$
H^{0}\left(\Sigma, \Omega_{Y \mid \Sigma}^{2}\right)=\{0\}
$$

Proof. With notations as in the previous proof, let $E$ be an effective divisor supported on the exceptional divisor of $\tau$, such that $-E$ is $\tau$-ample. Note that for a smooth surface $\Sigma \in|n H|$ contained in $Y_{\text {reg }}, \Sigma$ can be seen as a surface in the linear system $\left|n \tau^{*} H\right|$ on $\widetilde{Y}$ which does not intersect $E$ and it is equivalent to show that for some integer $l$, one has $H^{0}\left(\Sigma, \Omega_{\widetilde{Y}}^{2}(l E)_{\mid \Sigma}\right)=\{0\}$. As $-E$ is $\tau$-ample, for $n_{0}$ large enough, $n_{0} \tau^{*} H(-E)$ is ample, and thus, by vanishing on $\tilde{Y}$, we have for any $k \gg 0$

$$
H^{1}\left(\widetilde{Y}, \Omega_{\widetilde{Y}}^{2}\left(k E-k n_{0} \tau^{*} H\right)\right)=0
$$

For $n=k n_{0}$, let $\Sigma \in|n H|$ be contained in $Y_{\text {reg }}$, or equivalently $\Sigma \subset \widetilde{Y}, \Sigma \in$ $\left|\tau^{*} n H\right|$, not intersecting $E$. The vanishing (2.17) then implies that the restriction map

$$
H^{0}\left(\widetilde{Y}, \Omega_{\widetilde{Y}}^{2}(k E)\right) \rightarrow H^{0}\left(\Sigma, \Omega_{\widetilde{Y}}^{2}(k E)_{\mid \Sigma}\right)
$$

is surjective.

We now use [7, Thm.1.4] which implies that

$$
H^{0}\left(\widetilde{Y}, \Omega_{\widetilde{Y}}^{2}(k E)\right)=H^{0}\left(\widetilde{Y}, \Omega_{\widetilde{Y}}^{2}\right)
$$

for $k \geq 0$, because the singularities of $Y$ are canonical. By Lemma 2.14, we know that the right hand side is 0 , which concludes the proof.

\section{Application to Curve Classes}

This section is devoted to the proof of Theorem 1.4. The statement will first be reduced to the following Proposition 3.18, a variant of Proposition 1 in [27], which establishes this proposition in the case where $Y$ is smooth. We assume here that $Y$ is a Gorenstein projective threefold with trivial canonical bundle and canonical 
isolated singularities, such that $H^{1}\left(Y, \mathcal{O}_{Y}\right)=H^{2}\left(Y, \mathcal{O}_{Y}\right)=0$. Let $H$ be an ample line bundle on $Y$.

3.18. Proposition. Under these assumptions, there exists an integer $n_{0}$ such that for any multiple $n$ of $n_{0}$, a generic surface $\Sigma \subset Y$ in the linear system $|n H|$ has the following property: The integral cohomology $H^{2}(\Sigma, \mathbb{Z})$ is generated over $\mathbb{Z}$ by classes $\beta_{i}$ which become algebraic on some small deformation $\Sigma_{i}$ of $\Sigma$ in $Y$.

In this statement, a small deformation $\Sigma_{i}$ of $\Sigma$ in $X$ is a surface parameterized by a point in a small ball in $|n H|$ centered at the point parameterizing $\Sigma$. The family of surfaces parameterized by this ball is then topologically trivial, which makes the parallel transport of $\beta_{i}$ to a cohomology class on $\Sigma_{i}$ canonical.

We postpone the proof of this proposition, and prove now Theorem 1.4.

Proof of Theorem 1.4. Let $Y \subset X$ be as in the statement of Theorem 1.4. $Y$ is a local complete intersection and the canonical bundle $K_{Y}$ is trivial by adjunction, using the equality $\operatorname{det} \mathcal{E}=-K_{X}$. As $X$ is rationally connected, we have $H^{q}\left(X, \mathcal{O}_{X}\right)=0$ for $q \geq 1$. As $-K_{X}$ is 1 -ample, it follows that

$$
H^{1}\left(Y, \mathcal{O}_{Y}\right)=0,
$$

using the Sommese vanishing theorem [22, Proposition 1.14] and the Koszul resolution of $\mathcal{I}_{Y}$. As $Y$ has trivial canonical bundle, we then also have

$$
H^{2}\left(Y, \mathcal{O}_{Y}\right)=0
$$

by Serre's duality.

Let $H_{X}$ be an ample line bundle on $X$, and $H$ its restriction to $Y$. Let $n$ be chosen in such a way that the conclusion of Proposition 3.18 holds for the pair $(Y, H)$. Let $Z$ be a general member of $\left|n H_{X}\right|$. Consider the complete intersection surface

$$
\Sigma:=Z \cap Y \stackrel{j}{\hookrightarrow} X
$$

We claim that the Gysin map

$$
j_{*}: H^{2}(\Sigma, \mathbb{Z})=H_{2}(\Sigma, \mathbb{Z}) \rightarrow H_{2}(X, \mathbb{Z})=H^{2 n-2}(X, \mathbb{Z})
$$

is surjective. Indeed, we first make the remark that the restriction to a general sufficiently ample hypersurface of $X$ of a 1-ample vector bundle on $X$ is ample. As the chosen hypersurface $Z$ is general, we may thus assume that $\mathcal{E}_{\mid Z}$ is ample 
of rank $n-3$. But the surface $\Sigma \subset Z$ is the zero locus of a section of the ample vector bundle $\mathcal{E}_{\mid Z}$ of rank $n-3$ on $Z$, and $\operatorname{dim} Z=n-1$; Sommese's theorem [22, Proposition 1.16] extending Lefschetz hyperplane section theorem thus implies that the natural map

$$
j_{*}^{\prime}: H^{2}(\Sigma, \mathbb{Z})=H_{2}(\Sigma, \mathbb{Z}) \rightarrow H_{2}(Z, \mathbb{Z})
$$

is surjective, where $j^{\prime}$ is the inclusion of $\Sigma$ in $Z$. We finally apply the Lefschetz theorem on hyperplane sections to the inclusion $k: Z \hookrightarrow X$ to conclude that the Gysin map $k_{*}: H_{2}(Z, \mathbb{Z}) \rightarrow H_{2}(X, \mathbb{Z})$ is also surjective. Hence $j_{*}=k_{*} \circ j_{*}^{\prime}:$ $H_{2}(\Sigma, \mathbb{Z}) \rightarrow H_{2}(X, \mathbb{Z})$ is surjective, which proves the claim.

Let $\Sigma=Z \cap Y$ be as above and let $\beta \in H^{2 n-2}(X, \mathbb{Z})$. Then $\beta=j_{*} \alpha$, for some $\alpha \in H^{2}(\Sigma, \mathbb{Z}) . \quad Y$ has by assumption isolated canonical singularities, and the needed vanishings (3.19) and (3.20) hold. Thus we can apply Proposition 3.18. We thus can write $\beta=\sum_{i} \beta_{i}$, where $\beta_{i}$ has the property that its parallel transport $\beta_{i}^{\prime} \in H^{2}\left(\Sigma_{i}, \mathbb{Z}\right)$ is the class $\left[D_{i}\right]$ of a divisor $D_{i}$ on $\Sigma_{i}$, where $j_{i}: \Sigma_{i} \hookrightarrow X$ is a small deformation of $\Sigma$. We then have

$$
j_{*} \beta_{i}=j_{i *} \beta_{i}^{\prime}=j_{i *}\left(\left[D_{i}\right]\right)
$$

and thus the class $\beta$ can be written as $\sum_{i} j_{i *}\left(\left[D_{i}\right]\right)$, which is the class of the 1-cycle $\sum_{i} j_{i *}\left(D_{i}\right)$ of $X$.

It remains to prove Proposition 3.18. As already mentioned, the case where $Y$ is smooth is done in [27]. It is based on the study of the infinitesimal variation of the Hodge structure on $H^{2}(\Sigma, \mathbb{Z})$ and is in some sense purely local. However, we need to point out carefully the places where we use the assumptions on the singularities of $Y$, as the proposition is wrong if we only assume that $H^{i}\left(Y, \mathcal{O}_{Y}\right), i=1,2$ and $Y$ is Gorenstein with trivial canonical bundle. The simplest example is the case where $Y$ is a quintic hypersurface in $\mathbb{P}^{4}$ which is a cone over a smooth quintic surface $S$ in $\mathbb{P}^{3}$. Any smooth surface $\Sigma \subset Y_{\text {reg }}$ dominates $S$ via the projection $p$ from the vertex of the cone, and only those cohomology classes $\beta$ of degree 2 on $\Sigma$ which satisfy the property that $p_{*} \beta$ is of type $(1,1)$ on $S$ can become algebraic on a small deformation of $\Sigma$ in $Y$. Thus these classes do not generate $H^{2}(\Sigma, \mathbb{Z})$ since the Hodge structure on $H^{2}(S, \mathbb{Z})$ is non trivial. In this example, the singularities of $Y$ are isolated but not canonical and in fact the vanishing $H^{2}\left(\widetilde{Y}, \mathcal{O}_{\widetilde{Y}}\right)=0$ of Lemma 2.14 does not hold. 
For the convenience of the reader, we first summarize the strategy of the proof of Proposition 3.18, used in [27], which mainly consists to a reduction to Proposition 3.22 below.

Let $\Sigma \in|n H|$, and let us choose a small ball $U \subset|n H|$ centered at the point 0 parameterizing $\Sigma$. Let

$$
\pi: \mathcal{S} \rightarrow U, \mathcal{S} \subset U \times Y,
$$

be the restriction to $U$ of the universal family. This is a smooth projective map, and there is a variation of Hodge structures on the local system $R^{2} \pi_{*} \mathbb{Z}$, which is trivial since $U$ is simply connected.

Let $\mathcal{H}^{2}$ be the holomorphic vector bundle $R^{2} \pi_{*} \mathbb{Z} \otimes \mathcal{O}_{U}$. It is endowed with the Gauss-Manin connection $\nabla: \mathcal{H}^{2} \rightarrow \mathcal{H}^{2} \otimes \Omega_{U}$, and the Hodge filtration by holomorphic subbundles $F^{i} \mathcal{H}^{2}$. Furthermore, Griffiths transversality holds (cf. $[26$, I, 10.1.2]):

$$
\nabla\left(F^{i} \mathcal{H}^{2}\right) \subset F^{i-1} \mathcal{H}^{2} \otimes \Omega_{U}
$$

Denote by $F^{1} H^{2}$ the total space of $F^{1} \mathcal{H}^{2}$. The trivialisation of the local system $R^{2} \pi_{*} \mathbb{Z}$ on $U$ gives us a holomorphic map

$$
\Phi: F^{1} H^{2} \rightarrow H^{2}(\Sigma, \mathbb{C})
$$

which to a class $\alpha \in F^{1} H^{2}\left(\mathcal{S}_{t}\right)$ associates its parallel transport to $\mathcal{S}_{0}=\Sigma$.

We make four observations :

a) First of all, for the proof of 3.18 , we can work with cohomology $H^{2}(\Sigma, \mathbb{Z}) /$ torsion, because the torsion is made of classes of divisors.

b) Secondly, the set of classes $\beta_{t} \in H^{2}(\Sigma, \mathbb{Z}) /$ torsion which become algebraic (or equivalently of type $(1,1)$ ) on $\mathcal{S}_{t}$ for some $t \in U$ identifies (via the inclusion of $H^{2}(\Sigma, \mathbb{Z}) /$ torsion in $\left.H^{2}(\Sigma, \mathbb{C})\right)$ to the intersection of the image of $\Phi$ with $H^{2}(\Sigma, \mathbb{Z}) /$ torsion.

c) Consider the real part $F^{1} H_{\mathbb{R}}^{2}$ of $F^{1} H^{2}$, namely its intersection with the real vector bundle $H_{\mathbb{R}}^{2}$ with fiber $H^{2}\left(\mathcal{S}_{t}, \mathbb{R}\right)$ at $t \in U$. We clearly have $F^{1} H_{\mathbb{R}}^{2}=$ $\Phi^{-1}\left(H^{2}(\Sigma, \mathbb{R})\right)$.

d) Let us denote by $\Phi_{\mathbb{R}}$ the restriction of $\Phi$ to $F^{1} H_{\mathbb{R}}^{2}$. Then $\operatorname{Im} \Phi_{\mathbb{R}}$ is a cone.

By b) and c) above, what we want to prove is that the lattice $H^{2}(\Sigma, \mathbb{Z}) /$ torsion is generated over $\mathbb{Z}$ by points in $\operatorname{Im} \Phi_{\mathbb{R}} \cap H^{2}(\Sigma, \mathbb{Z}) /$ torsion. We use Lemma 3 
in [27], which says that given a lattice $L$, integral points of a cone in $L_{\mathbb{R}}$ with non-empty interior set generate $L$ over $\mathbb{Z}$. Using this and the above observation d), it suffices to prove that $\operatorname{Im} \Phi_{\mathbb{R}}$ has non-empty interior set and by observation c) and Sard's Lemma, it suffices to show that if $n$ is sufficiently divisible and $\Sigma$ is generic in $|n H|, \Phi$ is a submersion at some real point $\tilde{\lambda}$ in the fiber $F^{1} H_{\mathbb{R}, 0}^{2}$. Finally, as the set of points where $\Phi$ is a submersion is Zariski open, it suffices to show that if $n$ is sufficiently divisible, for $\Sigma$ generic in $|n H|, \Phi$ is a submersion at some point $\tilde{\lambda}$ (real or not) in the fiber $F^{1} H_{0}^{2}=F^{1} H^{2}(\Sigma, \mathbb{C}$ ).

We are now reduced to a statement involving the infinitesimal variations of Hodge structures on $H^{2}\left(\mathcal{S}_{t}\right)$ thanks to the following Lemma 3.21 (cf. [26, II, 5.3.4]):

Using transversality, the Gauss-Manin connection induces $\mathcal{O}_{U}$-linear maps

$$
\bar{\nabla}: F^{i} \mathcal{H}^{2} / F^{i-1} \mathcal{H}^{2} \rightarrow F^{i-1} \mathcal{H}^{2} / F^{i} \mathcal{H}^{2} \otimes \Omega_{U}
$$

whose fiber at $0 \in U$ gives for $i=1$ :

$$
\bar{\nabla}: H^{1}\left(\Sigma, \Omega_{\Sigma}\right) \rightarrow \operatorname{Hom}\left(T_{U, 0}, H^{2}\left(\Sigma, \mathcal{O}_{\Sigma}\right)\right),
$$

where $T_{U, 0}=H^{0}\left(\Sigma, n H_{\mid \Sigma}\right)$. We will use the same notation for the map obtained for $i=2$ :

$$
\bar{\nabla}: H^{0}\left(\Sigma, K_{\Sigma}\right) \rightarrow \operatorname{Hom}\left(T_{U, 0}, H^{1}\left(\Sigma, \Omega_{\Sigma}\right)\right),
$$

3.21. Lemma. Let $\tilde{\lambda} \in F^{1} H^{2}(\Sigma, \mathbb{C})$ project to $\lambda \in H^{1}\left(\Sigma, \Omega_{\Sigma}\right)=$ $F^{1} H^{2}(\Sigma, \mathbb{C}) / F^{2} H^{2}(\Sigma, \mathbb{C})$. Then $\Phi$ is a submersion at $\tilde{\lambda}$ if and only if $\bar{\nabla}(\lambda)$ is a surjective homomorphism from $T_{U, 0}$ to $H^{2}\left(\Sigma, \mathcal{O}_{\Sigma}\right)$.

Combining these facts, we see that Proposition 3.18 is a consequence of the following:

3.22. Proposition. Under the same assumptions as in Proposition 3.18, there exists an integer $n_{0}$ such that for any multiple $n$ of $n_{0}$, for generic $\Sigma \in|n H|$ and for generic $\lambda \in H^{1}\left(\Sigma, \Omega_{\Sigma}\right)$, the map $\bar{\nabla}(\lambda): H^{0}\left(\Sigma, \mathcal{O}_{\Sigma}(n H)\right) \rightarrow H^{2}\left(\Sigma, \mathcal{O}_{\Sigma}\right)$ is surjective.

This proposition, in the case where $Y$ is smooth, already appeared in [25], with a much simpler proof given in [27].

Note that because $K_{\widetilde{Y} \mid \Sigma}$ is trivial, one has by adjunction

$$
K_{\Sigma} \cong \mathcal{O}_{\Sigma}(n H)
$$


and the two spaces $H^{0}\left(\Sigma, \mathcal{O}_{\Sigma}(n H)\right), H^{2}\left(\Sigma, \mathcal{O}_{\Sigma}\right)$ are of the same dimension, and more precisely dual to each other, the duality being canonically determined by a choice of trivialization of $K_{\tilde{Y} \mid \Sigma}$.

It follows from formulas (3.27) and (3.29) given in Lemma 3.26 below that the homomorphisms $\bar{\nabla}(\lambda)$ are symmetric with respect to this duality. They can thus be seen as a system of quadrics on $H^{0}\left(\Sigma, \mathcal{O}_{\Sigma}(n H)\right)$, and the statement is that the generic one is non singular.

The proof of Proposition 3.22 in the smooth case uses Griffiths' theory which computes the Hodge filtration on the middle cohomology of a sufficiently ample hypersurface in a smooth projective variety and its infinitesimal variations, using residues of meromorphic forms. Following [9], the arguments can be made more formal and use only local infinitesimal data along the given hypersurface. But some needed vanishing statements still will use some global assumptions on the ambient variety. In fact, the main technical point that we will need and where we will actually use the assumptions on the singularities of $Y$ is Lemma 3.30 below.

Before stating it, let us recall a few points concerning infinitesimal variations of Hodge structures of hypersurfaces (surfaces in our case). Consider a smooth surface $\Sigma \subset Y$ in a linear system $|n H|$ on $Y$, where $H$ is ample on $Y$, and $Y$ is projective, smooth along $\Sigma$. There are two exact sequences deduced from the normal bundle sequence of $\Sigma$ in $Y$ (or rather $Y_{\text {reg }}$ ).

$$
\begin{gathered}
0 \rightarrow \Omega_{\Sigma}(n H) \rightarrow \Omega_{Y \mid \Sigma}^{2}(2 n H) \rightarrow K_{\Sigma}(2 n H) \rightarrow 0 \\
0 \rightarrow \mathcal{O}_{\Sigma} \rightarrow \Omega_{Y \mid \Sigma}(n H) \rightarrow \Omega_{\Sigma}(n H) \rightarrow 0 .
\end{gathered}
$$

Applying the long exact sequence associated to (3.23) provides us with a map

$$
\delta_{1}: H^{0}\left(\Sigma, K_{\Sigma}(2 n H)\right) \rightarrow H^{1}\left(\Sigma, \Omega_{\Sigma}(n H)\right) .
$$

The long exact sequence associated to (3.24) provides similarly a map

$$
\delta_{2}: H^{1}\left(\Sigma, \Omega_{\Sigma}(n H)\right) \rightarrow H^{2}\left(\Sigma, \mathcal{O}_{\Sigma}\right) .
$$

Set $\delta:=\delta_{2} \circ \delta_{1}: H^{0}\left(\Sigma, K_{\Sigma}(2 n H)\right) \rightarrow H^{2}\left(\Sigma, \mathcal{O}_{\Sigma}\right)$.

Similarly, let

$$
\delta^{\prime}: H^{0}\left(\Sigma, K_{\Sigma}(n H)\right) \rightarrow H^{1}\left(\Sigma, \Omega_{\Sigma}\right)
$$


be the map induced by the short exact sequence

$$
0 \rightarrow \Omega_{\Sigma} \rightarrow \Omega_{Y \mid \Sigma}^{2}(n H) \rightarrow K_{\Sigma}(n H) \rightarrow 0 .
$$

Let us recall the relevance of these maps to the study of infinitesimal variations of Hodge structures of the surfaces $\Sigma \subset Y$.

3.26. Lemma. We have for $u \in H^{0}\left(\Sigma, \mathcal{O}_{\Sigma}(n H)\right), \eta \in H^{0}\left(\Sigma, K_{\Sigma}\right)$

$$
\bar{\nabla}(\eta)(u)=\delta^{\prime}(\eta u),
$$

Furthermore, for $\sigma \in H^{0}\left(\Sigma, K_{\Sigma}(n H)\right), u, \eta$ as above, we have

$$
\bar{\nabla}\left(\delta^{\prime}(\sigma)\right)(u)=\delta(\sigma u) \text { in } H^{2}\left(\Sigma, \mathcal{O}_{\Sigma}\right) .
$$

Finally, for $\lambda \in H^{1}\left(\Sigma, \Omega_{\Sigma}\right)$, and any $u, \eta$ as above,

$$
\langle\bar{\nabla}(\lambda)(u), \eta\rangle=-\langle\lambda, \bar{\nabla}(\eta)(u)\rangle,
$$

where the first pairing uses Serre's duality between $H^{0}\left(\Sigma, K_{\Sigma}\right)$ and $H^{2}\left(\Sigma, \mathcal{O}_{\Sigma}\right)$, while the second pairing is the intersection pairing on $H^{1}\left(\Sigma, \Omega_{\Sigma}\right)$.

Proof. The two formulas (3.27) and (3.28) follow from Griffiths' general description of the maps $\bar{\nabla}$ acting on a given infinitesimal deformation $\rho(u) \in H^{1}\left(\Sigma, T_{\Sigma}\right)$ of $\Sigma$ (cf. [26, I, Theorem 10.21]), and from the fact that the exact sequences written above are all twists of the normal bundle exact sequence

$$
0 \rightarrow T_{\Sigma} \rightarrow T_{Y \mid \Sigma} \rightarrow \mathcal{O}_{\Sigma}(n H) \rightarrow 0
$$

which governs the Kodaira-Spencer map $\rho: H^{0}\left(\Sigma, \mathcal{O}_{\Sigma}(n H)\right) \rightarrow H^{1}\left(\Sigma, T_{\Sigma}\right)$.

Formula (3.29) is proved in [26, II, 5.3.3], (this is formula (5.14) there).

3.30. Lemma. Assume that $n$ is sufficiently large and that $\Sigma$ satisfies

$$
H^{0}\left(\Sigma, \Omega_{Y \mid \Sigma}^{2}\right)=0 .
$$

Then $\delta$ is surjective.

In particular, under the assumptions of Proposition 3.18, the map $\delta$ is surjective for $n$ sufficiently divisible.

Proof. The second statement follows indeed from the first, since we proved in Lemma 2.15 that when $Y$ has isolated canonical singularities and $H^{2}\left(Y, \mathcal{O}_{Y}\right)=0$, we have the vanishing (3.31) for $n$ sufficiently divisible. 
We first prove that $\delta_{1}$ is surjective for large $n$ and for any $\Sigma$ as above, without any further assumption on $Y$. Indeed, looking at the long exact sequence associated to (3.23), we find that the cokernel of $\delta_{1}$ is contained in $H^{1}\left(\Sigma, \Omega_{Y \mid \Sigma}^{2}(2 n H)\right)$. Consider the following exact sequence, where $\Omega_{Y}^{2}$ is defined as $i_{*} \Omega_{Y, r e g}^{2}, i: Y_{\text {reg }} \rightarrow Y$ being the inclusion of the regular locus:

$$
0 \rightarrow \Omega_{Y}^{2}(n H) \rightarrow \Omega_{Y}^{2}(2 n H) \rightarrow \Omega_{Y \mid \Sigma}^{2}(2 n H) \rightarrow 0 .
$$

We get by Serre's vanishing on $Y$ that for large $n$, both $H^{1}\left(Y, \Omega_{Y}^{2}(2 n H)\right)$ and $H^{2}\left(Y, \Omega_{Y}^{2}(n H)\right)$ vanish and it follows that $H^{1}\left(\Sigma, \Omega_{Y \mid \Sigma}^{2}(2 n H)\right)$ also vanishes.

It remains to prove that $\delta_{2}$ is surjective under assumption (3.31), for large $n$. Looking at the long exact sequence associated to (3.24), we find that the cokernel of $\delta_{2}$ is contained in $H^{2}\left(\Sigma, \Omega_{Y \mid \Sigma}(n H)\right)$. As $K_{\Sigma}=\left.K_{Y}(n H)\right|_{\Sigma}$ by adjunction, we find that $H^{2}\left(\Sigma, \Omega_{Y \mid \Sigma}(n H)\right)$ is Serre dual to

$$
H^{0}\left(\Sigma, T_{Y \mid \Sigma}\left(K_{Y \mid \Sigma}\right)\right)=H^{0}\left(\Sigma, \Omega_{Y \mid \Sigma}^{2}\right)
$$

which vanishes by assumption.

For the convenience of the reader, we now summarize the main steps in the proof of Proposition 3.22 given in [27] in the case where $Y$ is smooth with trivial canonical bundle, in order to make clear why the argument still works in the singular case, when $Y$ has isolated canonical singularities.

Let $n$ be divisible enough so that the conclusion of Lemma 2.15 holds. For any smooth $\Sigma \in|n H|$, we have the system $Q_{\Sigma}$ of quadrics on $V_{\Sigma}:=H^{0}\left(\Sigma, K_{\Sigma}\right) \cong$ $H^{0}\left(\Sigma, \mathcal{O}_{\Sigma}(n H)\right)$ given as the image of $H^{1}\left(\Sigma, \Omega_{\Sigma}\right)$ in $S^{2} H^{0}\left(\Sigma, \mathcal{O}_{\Sigma}(n H)\right)^{*}$, via the map

$$
q: H^{1}\left(\Sigma, \Omega_{\Sigma}\right) \rightarrow S^{2} H^{0}\left(\Sigma, \mathcal{O}_{\Sigma}(n H)\right)^{*}, \quad \lambda \mapsto q_{\lambda}
$$

where

$$
q_{\lambda}(\eta, u):=<\lambda, \bar{\nabla}(\eta)(u)>
$$

for $\eta \in H^{0}\left(\Sigma, K_{\Sigma}\right)=H^{0}\left(\Sigma, \mathcal{O}_{\Sigma}(n H)\right), u \in H^{0}\left(\Sigma, \mathcal{O}_{\Sigma}(n H)\right)$. As explained above, and using (3.29), the statement to be proved can be restated by saying that for generic $\Sigma, Q_{\Sigma}$ contains a smooth quadric. We will apply the following lemma (cf. [27, Proposition 1.10], [25, Lemma 15]) which is an easy consequence of Bertini's Lemma: 
3.33. Lemma. A linear system of quadrics $Q$ on a vector space $V$ contains a smooth member if the following condition (*) holds:

$\left(^{*}\right)$ Let $B \subset \mathbb{P}(V)$ be the base locus of $Q$ and $b:=\operatorname{dim} B$. Then for any $k \leq b$, the subset $W_{k} \subset \mathbb{P}(V)$, defined as

$$
W_{k}:=\left\{v \in B, i_{v}: Q \rightarrow V^{*} \text { has rank } \leq k\right\}
$$

has dimension $<k$.

(In this statement, the map $i_{v}$ associated to $v$ is constructed by contraction.) For example, if $b=0$, the base-locus consists of isolated points, and by Bertini, if any member of $Q$ was singular, it would be singular at some common point $v \in B$, which would satisfy $i_{v}=0$, which is excluded by assumption $\left(^{*}\right)$.

The first step in the proof of the proposition is the following asymptotic upperbound for the dimension of the base-locus of $Q_{\Sigma}$ for generic $\Sigma \in|n H|$ as a function of $n$.

3.34. Lemma. There exists a positive number $c$, such that for any $n$ and for generic $\Sigma \in|n H|$, the dimension of the base-locus of $Q_{\Sigma}$ is $\leq \mathrm{cn}^{2}$.

The proof of this lemma is based on the following arguments, none involving the smoothness of $Y$ away from $\Sigma$.

The first argument is Proposition 1.6 in [25], which concerns degenerations of $\Sigma$ to a surface $\Sigma_{0}$ with nodes $p_{1}, \ldots, p_{N}$ : that is we have a smooth family of surfaces $\mathcal{S} \rightarrow \Delta, \mathcal{S} \subset \Delta \times Y$, with central fiber isomorphic to $\Sigma_{0}$.

3.35. Lemma. The limiting linear system $Q_{\Sigma_{0}}$ of quadrics on $V_{\Sigma_{0}}=$ $H^{0}\left(\Sigma_{0}, \mathcal{O}_{\Sigma_{0}}(n H)\right)$ contains the quadrics $q_{i} \in S^{2} V_{\Sigma_{0}}^{*}$ defined (up to a coefficient depending on the trivialisation of $\left.\mathcal{O}_{p_{i}}(2 n H)\right)$ by evaluation at $p_{i}$ :

$$
q_{i}(\eta)=\eta^{2}\left(p_{i}\right), \forall \eta \in H^{0}\left(\Sigma_{0}, \mathcal{O}_{\Sigma_{0}}(n H)\right) .
$$

The second argument is then provided by the construction of surfaces $\Sigma_{0}$ with many nodes, imposing many conditions on $V_{\Sigma_{0}}=H^{0}\left(\Sigma_{0}, \mathcal{O}_{\Sigma_{0}}(n H)\right)$. Such surfaces $\Sigma_{0}$ are obtained in [27] as discriminant loci for symmetric matrices of size $(n, n)$ with coefficients in $H^{0}(Y, H)$ (where $H$ is supposed to be very ample on $Y)$. Using results of Barth in [2], such a generically chosen discriminant surface has only ordinary double points, whose number $N$ is a cubic polynomial in $n$, 
(precisely equal to $\left(\begin{array}{c}n+1 \\ 3\end{array}\right) H^{3}$ ), hence with leading term $\frac{n^{3}}{6} H^{3}$. Notice that, by Riemann-Roch, $H^{0}\left(\Sigma_{0}, \mathcal{O}_{\Sigma_{0}}(n H)\right)$ has dimension given by a cubic polynomial in $n$ with leading term also equal to $\frac{n^{3}}{6} H^{3}$. Finally, we prove in [27], by considering a natural resolution of the ideal sheaf of subset $W=\left\{p_{1}, \ldots, p_{N}\right\} \subset \Sigma_{0}$ of nodes of $\Sigma_{0}$, that $W$ imposes independent conditions to the linear system $\left|\mathcal{O}_{Y}((n+2) H)\right|$, and it follows easily that for some constant $c>0$

$$
\operatorname{dim} H^{0}\left(Y, \mathcal{I}_{W}(n H)\right) \leq c n^{2} .
$$

The proofs given there do not involve the smoothness of $Y$, as long as $\Sigma_{0}$ does not meet the singular locus of $Y$.

By Lemma 3.35, the base locus of the limiting system of quadrics $Q_{\Sigma_{0}}$ on $V_{\Sigma_{0}}$ is contained in $\mathbb{P}\left(H^{0}\left(\Sigma_{0}, \mathcal{I}_{W}(n H)\right)\right)$ so that (3.36) completes the proof of Lemma 3.34 .

We want next to study the sets $W_{\Sigma, k}$ introduced in Lemma 3.33, for generic $\Sigma \in|n H|$. By Lemma 3.34, we can restrict to the range $k \leq c n^{2}$. The second step in the proof is thus the study of the locus $W_{c, \Sigma} \subset \mathbb{P}\left(V_{\sigma}\right)$ defined as

$$
W_{c n^{2}, \Sigma}:=\left\{v \in \mathbb{P}\left(V_{\Sigma}\right), i_{v}: Q_{\Sigma} \rightarrow V_{\Sigma}^{*} \text { has rank } \leq c n^{2}\right\}
$$

and we need to prove the following lemma (cf. Lemma 12 in [27]):

3.37. Lemma. There exists a constant $A$ independent of $n$, such that for generic $\Sigma \in|n H|$, one has $\operatorname{dim} W_{c n^{2}, \Sigma} \leq A$.

The proof of this statement given in loc. cit. does not involve the smoothness of the ambient space $Y$. It is obtained by studying the case where $\Sigma$ is of Fermat type. Namely, one chooses a generic projection $p: Y \rightarrow \mathbb{P}^{3}$ such that $p^{*} \mathcal{O}_{\mathbb{P}^{3}}(1)=$ $H$, and one takes for $\Sigma$ the inverse image of the Fermat surface $S_{n}$ of degree $n$ in $\mathbb{P}^{3}$. One is led to compare the infinitesimal variation of Hodge structure for $\Sigma$ with a sum of twisted infinitesimal variations of Hodge structure for $S_{n}$. This comparison involves only the map $p: \Sigma \rightarrow S_{n}$ and the restriction of $\Omega_{Y}$ to $\Sigma$, and not the geometry of $Y$ away from $\Sigma$.

Using Lemma 3.37, we conclude that all the sets $Z_{k, \Sigma}$ introduced in Lemma 3.33 have dimension $\leq A$, and in order to check the criterion given in Lemma 3.33, it suffices now to study the following sets $W_{A, \Sigma}$ :

$$
W_{A, \Sigma}:=\left\{v \in \mathbb{P}\left(V_{\Sigma}\right), i_{v}: Q_{\Sigma} \rightarrow V_{\Sigma}^{*} \text { has rank } \leq A\right\} .
$$


This is the third step and last step of the proof:

3.38. Lemma. Let $H$ be normally generated on $Y$ and let $A$ be a given (large) constant. Then for $n>A$, and for smooth $\Sigma \in|n H|$, the set $W_{A, \Sigma}$ is empty.

Proof. The proof is by contradiction. Let $v \in W_{A, \Sigma}$ and let $M \subset H^{0}\left(\Sigma, \mathcal{O}_{\Sigma}(2 n H)\right)$ be the kernel of the composite map:

$$
H^{0}\left(\Sigma, \mathcal{O}_{\Sigma}(2 n H)\right) \stackrel{\delta^{\prime}}{\rightarrow} H^{1}\left(\Sigma, \Omega_{\Sigma}\right) \stackrel{i_{v} \circ q}{\longrightarrow} H^{0}\left(\Sigma, K_{\Sigma}\right)^{*},
$$

where the map $\delta^{\prime}: H^{0}\left(\Sigma, \mathcal{O}_{\Sigma}(2 n H)\right) \rightarrow H^{1}\left(\Sigma, \Omega_{\Sigma}\right)$ is defined in (3.25) and $q$ is defined in (3.32). As the rank of $i_{v} \circ q$ is $\leq A$, one has codim $M \leq A$. Identifying $H^{0}\left(\Sigma, K_{\Sigma}\right)^{*}$ with $H^{2}\left(\Sigma, \mathcal{O}_{\Sigma}\right)$, and using formula (3.28) of Lemma 3.26, one sees on the other hand that $M$ is the kernel of the composite map:

$$
H^{0}\left(\Sigma, \mathcal{O}_{\Sigma}(2 n H)\right) \stackrel{v}{\rightarrow} H^{0}\left(\Sigma, \mathcal{O}_{\Sigma}(3 n H)\right) \stackrel{\delta}{\rightarrow} H^{2}\left(\Sigma, \mathcal{O}_{\Sigma}\right),
$$

where $v$ is multiplication by $v$.

It also follows from the combination of the three formulas given in Lemma 3.26 that for $v, v^{\prime} \in H^{0}\left(\Sigma, \mathcal{O}_{\Sigma}(n H)\right)$, and for any $m \in H^{0}\left(\Sigma, \mathcal{O}_{\Sigma}(2 n H)\right)$, we have

$$
\left\langle v, \delta\left(v^{\prime} m\right)\right\rangle=\left\langle v^{\prime}, \delta(v m)\right\rangle
$$

where $\langle$,$\rangle is the Serre pairing between H^{0}\left(\Sigma, \mathcal{O}_{\Sigma}(n H)\right)=H^{0}\left(\Sigma, K_{\Sigma}\right)$ and $H^{2}\left(\Sigma, \mathcal{O}_{\Sigma}\right)$. Taking $m \in M$, so that $\delta(m v)=0$, we deduce from (3.39) that $\delta\left(H^{0}\left(\Sigma, \mathcal{O}_{\Sigma}(n H)\right) \cdot M\right)$ is orthogonal to $v$ with respect to Serre duality. But this provides a contradiction by the following argument:

First of all, $M$ has no base point. Indeed, from the exact sequence (3.25), we see that $M$ contains the image of $H^{0}\left(\Sigma, \Omega_{Y \mid \Sigma}^{2}(n H)\right)$ in $H^{0}\left(\Sigma, \mathcal{O}_{\Sigma}(2 n H)\right)$. As $n$ is large, $\left.\Omega_{Y \mid \Sigma}^{2}(n H)\right)$ is globally generated and its image in $H^{0}\left(\Sigma, \mathcal{O}_{\Sigma}(2 n H)\right)$ thus generates $\mathcal{O}_{\Sigma}(2 n H)$ at any point. Thus we can apply the following result due to Green [8] to the linear system $M$ on $\Sigma$.

3.40. Proposition. Let $Z$ be any projective manifold and $H$ be a very ample normally generated line bundle on $Z$. Let $A$ be a given constant, and for $m>A$, let $K \subset H^{0}\left(Z, \mathcal{O}_{Z}(m H)\right)$ be a subspace of codimension $\leq A$. Then

$$
H^{0}\left(Z, \mathcal{O}_{Z}(H)\right) \cdot K \subset H^{0}\left(Z, \mathcal{O}_{Z}((m+1) H)\right)
$$

has codimension $\leq A$, with strict inequality if $K$ has no base-point and $K \neq$ $H^{0}\left(Z, \mathcal{O}_{Z}(H)\right)$. 
As $M$ has no base point and $2 n>A$, we conclude that the codimensions of the subspaces $H^{0}\left(\Sigma, \mathcal{O}_{\Sigma}(k H)\right) \cdot M \subset H^{0}\left(\Sigma, \mathcal{O}_{\Sigma}((2 n+k) H)\right)$ are strictly decreasing until they fill-in $H^{0}\left(\Sigma, \mathcal{O}_{\Sigma}((2 n+k) H)\right)$. As codim $M \leq A<n$, we conclude that $H^{0}\left(\Sigma, \mathcal{O}_{\Sigma}(n H)\right) \cdot M=H^{0}\left(\Sigma, \mathcal{O}_{\Sigma}(3 n H)\right)$. But then we use Lemma 3.30 saying that $\delta$ is surjective. It thus follows that

$$
\delta\left(H^{0}\left(\Sigma, \mathcal{O}_{\Sigma}(n H)\right) \cdot M\right)=H^{2}\left(\Sigma, \mathcal{O}_{\Sigma}\right),
$$

which contradicts the fact that $\delta\left(H^{0}\left(\Sigma, \mathcal{O}_{\Sigma}(n H)\right) \cdot M\right)$ is orthogonal to $v$ with respect to Serre duality.

\section{REFERENCES}

[1] Atiyah, M.F. And Hirzebruch, F. Analytic cycles on complex manifolds, Topology 1, 25-45 (1962).

[2] W. Barth. Counting singularities of quadratic forms on vector bundles, in Vector bundles and Differential Equations, Proceedings, Nice 1979, A. Hirschowitz Ed, Progress in Mathematics 7, Birkhäuser (1980).

[3] Demailly, Jean-Pierre, Multiplier ideal sheaves and analytic methods in algebraic geometry, School on Vanishing Theorems and Effective Results in Algebraic Geometry (Trieste, 2000), (2001).

[4] J.-P. Demailly, T. Peternell, M. Schneider. Compact Kähler manifolds with Hermitian semipositive anticanonical bundle, Compositio. Math 101, 217-224 (1996).

[5] Floris, Enrica. Fundamental divisors on Fano varieties of index $n-3$, arXiv: 1009.0812, 2010.

[6] T. Graber, J. Harris and J. Starr, Families of rationally connected varieties. J. Amer. Math. Soc., 16(1), 57-67 (2003).

[7] Daniel Greb, Stefan Kebekus, Sandor J. Kovács, Thomas Peternell. Differential Forms on Log Canonical Spaces, arXiv:1003.2913.

[8] M. Green. Restriction of linear series to hyperplanes and some results of Macaulay and Gotzmann, in Algebraic curves and projective geometry (Trento 1988), Lecture Notes in Math. 1389, Springer, Berlin 1989.

[9] M. Green. The period map for hypersurface sections of high degree of an arbitrary variety, Compositio Math. 55 (1985), no. 2, 135-156.

[10] Yujiro Kawamata. On effective non-vanishing and base-point-freeness. Asian J. Math., 4(1):173-181, 2000. Kodaira's issue.

[11] János Kollár. Singularities of pairs. In Algebraic geometry - Santa Cruz 1995, volume 62 of Proc. Sympos. Pure Math., pages 221-287. Amer. Math. Soc., Providence, RI, 1997.

[12] Kollár, J. Lemma p. 134 in Classification of irregular varieties, edited by E. Ballico, F. Catanese, C. Ciliberto, Lecture Notes in Math. 1515, Springer (1990).

[13] J. Kollár, Y. Miyaoka and S. Mori, Rationally connected varieties, Journal of Algebraic Geometry 1, 429-448, (1992). 
[14] János Kollár. Rational curves on algebraic varieties, volume 32 of Ergebnisse der Mathematik und ihrer Grenzgebiete. 3. Folge. A Series of Modern Surveys in Mathematics. Springer-Verlag, Berlin, 1996.

[15] J. Kollár. Holomorphic and pseudo-holomorphic curves on rationally connected varieties, Portugaliae Mathematica, Volume 67, Issue 2, (2010) 155-179.

[16] Massimiliano Mella. Existence of good divisors on Mukai varieties. J. of Algebraic Geometry, 8, No.2, 197-206, 1999.

[17] Yoichi Miyaoka. The Chern classes and Kodaira dimension of a minimal variety. In Algebraic geometry, Sendai, 1985, volume 10 of Adv. Stud. Pure Math., pages 449-476. North-Holland, Amsterdam, 1987.

[18] Thomas Peternell. Varieties with generically nef tangent bundles. arXiv, 0807.0982, 2008.

[19] Miles Reid. Projective morphisms according to Kawamata. 1983.

[20] B. Saint-Donat. Projective models of $K-3$ surfaces. Amer. J. Math., 96:602-639, 1974.

[21] V. V. Škurov. Smoothness of a general anticanonical divisor on a Fano variety. Izv. Akad. Nauk SSSR Ser. Mat., 43(2):430-441, 1979.

[22] A. Sommese, Submanifolds of abelian varieties, Math. Ann. 233 (1978), 229-256.

[23] C. Soulé, C. Voisin. Torsion cohomology classes and algebraic cycles on complex projective manifolds, Adv. Math. 198 (2005), no. 1, 107-127.

[24] J. Starr. A pencil of Enriques surfaces of index one with no section, Algebra Number Theory 3 (2009), no. 6, 637-652.

[25] C. Voisin. Densité du lieu de Noether-Lefschetz pour les sections hyperplanes des variétés de Calabi-Yau de dimension 3, Internat. J. Math. 3 (1992), no. 5, 699-715.

[26] C. Voisin. Hodge theory and complex algebraic geometry I and II, Cambridge Studies in Advanced Mathematics 76, 77, Cambridge University Press, Cambridge (2002, 2003).

[27] C. Voisin, On integral Hodge classes on uniruled and Calabi-Yau threefolds, in Moduli Spaces and Arithmetic Geometry, Advanced Studies in Pure Mathematics 45, pp. 43-73, (2006).

[28] Qi Zhang. On projective varieties with nef anticanonical divisors, Math. Ann. 332, 697-703 (2005).

Andreas Höring

Université Pierre et Marie Curie and Albert-Ludwig Universität Freiburg,

TGA Case 247, 4 place Jussieu, 75005 Paris, France

E-mail: hoering@math.jussieu.fr

Claire Voisin

CNRS, Université Pierre et Marie Curie,

Institut de Mathématiques de Jussieu,

TGA Case 247, 4 place Jussieu, 75005 Paris, France

E-mail: voisin@math.jussieu.fr 